\title{
DIGITAL IMAGE CORRELATION FROM COMMERCIAL TO FOS SOFTWARE: A MATURE TECHNIQUE FOR FULL-FIELD DISPLACEMENT MEASUREMENTS
}

\author{
V. Belloni ${ }^{\mathrm{a} *}$ R. Ravanelli ${ }^{\mathrm{a}}$ A. Nascetti ${ }^{\mathrm{a}, \mathrm{b}}$, M. Di Rita ${ }^{\mathrm{a}}$, D. Mattei $^{\mathrm{c}}$, M. Crespi $^{\mathrm{a}}$ \\ ${ }^{a}$ Geodesy and Geomatics Division, DICEA - University of Rome "La Sapienza”, Rome, Italy \\ $<$ valeria.belloni, roberta.ravanelli, martina.dirita, mattia.crespi $>@$ uniroma1.it \\ ${ }^{\mathrm{b}}$ Geoinformatics, KTH Royal Institute of Technology, Stockholm, Sweden \\ $<$ nascetti $>@$ kth.se \\ ${ }^{\mathrm{c}}$ Department of Structural and Geotechnical Engineering - University of Rome La Sapienza, Rome, Italy
}

Commission II WG II/7

\begin{abstract}
KEY WORDS: Close range photogrammetry, Digital Image Correlation, Full-field displacement measurement, Commercial and Free
\end{abstract} and Open Source Software

\begin{abstract}
:
In the last few decades, there has been a growing interest in studying non-contact methods for full-field displacement and strain measurement. Among such techniques, Digital Image Correlation (DIC) has received particular attention, thanks to its ability to provide these information by comparing digital images of a sample surface before and after deformation. The method is now commonly adopted in the field of civil, mechanical and aerospace engineering and different companies and some research groups implemented 2D and 3D DIC software. In this work a review on DIC software status is given at first. Moreover, a free and open source 2D DIC software is presented, named py2DIC and developed in Python at the Geodesy and Geomatics Division of DICEA of the University of Rome "La Sapienza"; its potentialities were evaluated by processing the images captured during tensile tests performed in the Structural Engineering Lab of the University of Rome "La Sapienza" and comparing them to those obtained using the commercial software Vic2D developed by Correlated Solutions Inc, USA. The agreement of these results at one hundredth of millimetre level demonstrate the possibility to use this open source software as a valuable 2D DIC tool to measure full-field displacements on the investigated sample surface.
\end{abstract}

\section{INTRODUCTION}

Traditionally, in the field of structural engineering, conventional contact measurement techniques, such as accelerometers and strain gauges, are used to measure strain and displacement. However, these conventional sensors can only provide measurements at the location of the trasducers, they need wiring and may induce mass loading (Baqersad et al., 2017). Besides, they require a complex setup which complicates the measurement of displacements and strains outside laboratory conditions. It is evident how the noncontact measurement techniques, which do not require the use of dedicated devices, can play an important role in such a context.

The full-field non-contact optical methods include both interferometric and non-interferometric techniques. The interferometric metrologies measure the deformation by recording the phase difference of the scattered light wave from the test object surface before and after deformation (Pan et al., 2009). Holography interferometry, speckle interferometry and moiré interferometry fall into this category.

On the other hand, non-interferometric techniques compute the deformation by comparing the gray-scale variations of the object surface during the deformation process. Digital Image Correlation (DIC) is the most popular non-interferometric technique.

The DIC method was first developed by a group of researchers of the University of South Carolina in the 1980s (W. H. Peters, 1982). From then on, it has received attention thanks to the recent advances in the field of low-cost, high resolution digital cameras, computer power and memory storage and its capability to achieve full-field measurements (Baqersad et al., 2017). As a matter of

* Corresponding author fact, DIC can be used to measure full-field displacements and strains of different materials or structures subjected to incremental loads by comparing digital images in the un-deformed and deformed states. It is based on the template matching, a method generally used in photogrammetry and computer vision to retrieve homologous points (Ravanelli et al., 2017).

DIC compares a series of images of a sample at different stages of deformation, follows the pixel movement in the Area of Interest (AOI) and calculates displacement and strain by tracking (or matching) the same points between the recorded images (McCormick and Lord, 2010).

The two-dimensional (2D) DIC method requires a single fixed camera to record the digital images of the sample surface during the deformation process. The typical image acquisition system is composed by a planar sample connected to a mechanical loading, a digital camera that records images and a computer to process the acquired images. The method is used to estimate in-plane displacements and strains of the planar sample surface. The threedimensional (3D) DIC technique is instead adopted to measure 3D displacement fields and it is based on the principle of binocular stereovision that is to say, on the use of two stereo cameras whose positions is fixed in place when the images are simultaneously acquired (Pan et al., 2009).

Alternatively, the constraint about the fixed position of the camera can be relaxed, but the investigated surface must be firmly linked to a $2 \mathrm{D}$ reference frame (e.g. materialized with a suitable textured frame surrounding the investigated surface).

Nowadays, there are various 2D and 3D DIC commercial software available on the market that can be used to evaluate strains and displacements in civil, mechanical and aerospace applica- 
tions. Although the software are fast and robust, their limitation is their inherent cost. Furthermore, some restrictions are imposed to users because no customizations of the source code, to better fit their requirements, are allowed. Alternatively, open source, user friendly software can remarkably reduce costs and can be tailored to user needs (Harilal Ramesh and Ramji M, 2014). For all these reasons, some research DIC codes were implemented and made freely available to the scientific community. Anyway, some of them are implemented in Matlab language (e.g. (Jones et al., 2014)), for which the purchase of a license is however necessary.

Among these research DIC software, a new, free and open source software (FOSS) for 2D DIC, named py2DIC, was developed at the Geodesy and Geomatics Division of the University of Rome "La Sapienza". It was developed in Python and the source code is freely available at https://github.com/Geod-Geom/py2DIC.

Specifically, the aim of this work is to investigate the py2DIC potentialities by comparing its performances with those of a wellestablished software. In detail, in Section 2 a description of the available commercial and open source software is provided; Section 3 illustrates the features of the developed software and Section 4 focuses on the first comparison between py2DIC and the commercial software Vic-2D. In the end, in Section 6, some conclusions are drawn and future prospects are outlined.

\section{COMMERCIAL DIC SOFTWARE AND RESEARCH DIC CODES}

Nowadays, different companies provide 2D and 3D DIC commercial software to estimate displacements and strain fields. Besides, some research DIC codes are now freely available to the scientific community.

\subsection{Commercial software}

Among software companies, Correlated Solutions, CorreliSTC, Dantec Dynamics, Gom, Image Systems, Imetrum, LaVision and Match ID are the most important DIC software vendors. They provide high cost instruments to perform 2D and 3D DIC measurements; many of them offer both hardware and software. They invest in developing and upgrading their software version in order to produce fast and robust software characterized by high accuracy and user friendliness.

Correlated Solutions, Inc., was founded in 1998 in order to commercialize DIC and it is recognized as world leader and the inventor of this technology. It offers user friendly commercial software, named Vic, based on optimized correlation algorithms to provide 2D and 3D full-field displacement and strain covering a wide range of applications in different sector from automotive to biological (Correlated Solutions, 2018)

CorreliSTC developed a licensed DIC software from the Laboratory of Mechanics and Technology of ENS Cachan following the specifications defined by Airbus Group Innovations. It can be used to measure 2D and 3D DIC displacements and strains in the aeronautics, automotive and energy industrial sectors (CorreliSTC, 2018).

Dantec Dynamics was founded during the 1950s and it is specialized in instrumentation for flow measurement and particle characterisation. Among the optical measurement systems it supports, it offers a software named Q-400 to perform 2D and 3D DIC measurements (Dantec Dynamics, 2018).
Gom is an industrial manufacturer specialized in developing, producing and distributing optical measurement solutions and technologies for 3D coordinate measurement and deformation analysis. Among the developed technologies, ARAMIS is a noncontact and material-independent measuring system which provides solution for full-field analysis (gom, 2018).

Image Systems was born in 1999 and it offers a software named TEMA Stereo DIC which allows for 2D and 3D measuring of full-field displacements and strains (Image Systems, 2018).

Imetrum is a company composed by a group of experts in noncontact measurements who operate in the field of rail bridge, road, geotechnical and structural monitoring. It offers the 3D PDT (Precision Displacement Trackers), a powerful, camera-based displacement sensor useful to obtain full-field 3D displacements (Imetrum, 2018).

LaVision was founded in 1989 as a spin-off from Max Planck Institute and Laser Laboratory in Gottingen and it is focused on imaging systems, smart optical sensors, measurement technologies and software tools. It offers the 2D and 3D DIC system StrainMaster, a non-intrusive optical tool for shape and deformation analysis which combines the DIC algorithms with high quality hardware to offer an easy to use instrument for materials analysis (LaVision, 2018).

Finally, MatchID was founded by experts in the field of image correlation and it offers 2D and stereo DIC software (MatchID, 2018).

\subsection{Research DIC codes}

Regards the free and open source software, Ncorr, Digital Image Correlation Engine (DICe) and Yet Another Digital Image Correlation Software (YADICS) are among the most popular ones.

Ncorr is a free and open source 2D subset-based DIC software developed at the Georgia Institute of Technology. It is implemented in Matlab but compute intensive algorithms are written in $\mathrm{C}++$ and incorporated into Matlab to reach a greater efficiency (Blaber et al., 2015). It is characterized by a Graphical User Interface (GUI) written mostly in m-code and it provides plotting tools for figure creation (Ncorr, 2018). Ncorr was developed on Windows 7, it has also been tested on Ubuntu 14.04LTS but not on Mac OS. The software is able to estimate 2D full-field displacement and strain starting from a set of speckle images and it suits a wide range of applications. Not only typical solid mechanics tests but also geotechnical and biomedical experiments were performed to verify the potentialities of the Ncorr algorithms.

DICe is an open source DIC tool developed at the Sandia National Laboratories. It is written in $\mathrm{C}++$ and it is provided with a GUI implemented with html, css, and javascript. It is cross-platform (Windows, Linux, and Mac) and it can be used for 2D and stereo DIC analysis (Turner, 2015).

Finally, YADICS was developed at the Laboratoire de Mécanique de Lille (LML) and runs on a linux operating system. It is implemented in $\mathrm{C}++$ and it is used for 2D and 3D solid and fluid kinematics field measurements (Seghir and Coudert, 2014).

\section{PY2DIC}

py2DIC is a 2D DIC software developed at the Geodesy and Geomatics Division of DICEA of the University of Rome "La Sapienza" (Belloni, 2017). It is a FOSS and it is completely 
written in Python, a free, open source, cross-platform and easy to use programming language. It leverages the potentialities of OpenCV, an open source computer vision and machine learning software library (Bradski and Kaehler, 2008) and it is provided with a GUI. It is cross-platform and it has been tested on Windows, Linux, and Mac.

The software is able to compare two or more images of a sample surface acquired at different stages of deformation and it returns full-field displacement and strain maps.

It is based on the template matching method, the well-known technique for matching patterns on the basis of the cross-correlation coefficient.

The implemented 2D DIC procedure consists of several steps. First of all, the AOI of the reference image is divided into a uniformly spaced grid. Then, a portion of the reference image (the template) is compared to a corresponding larger subset (the search window) of the search image using a convolution procedure. Specifically, the search window in the search image is opened exactly around the central pixel $p(x, y)$ of the template. The method implemented in py2DIC is represented in Figure 1.

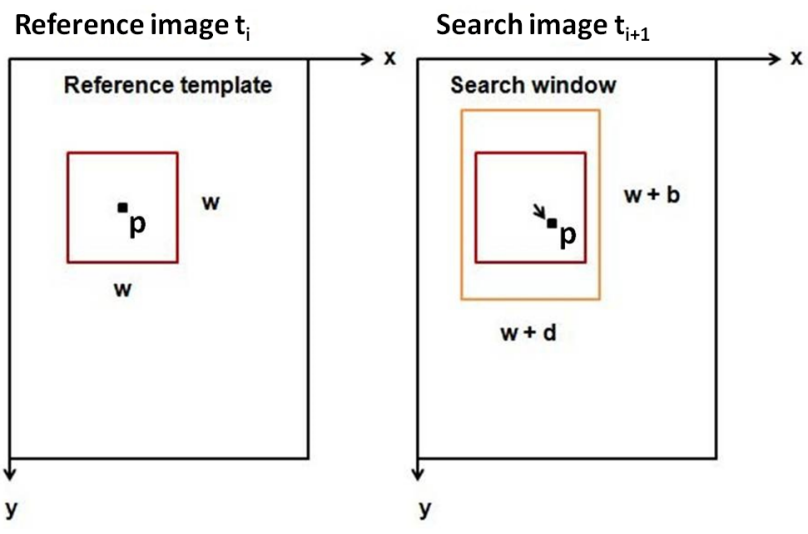

Figure 1: Scheme of the image pairs together with the reference template, the central pixel and the search window.

As shown in Figure 1, the size of the reference template is $(w \times w)$ and the size of the search window is $(w+d) \times(w+b)$, where $w$ is the template width and $d$ and $b$ are the edge along the $x$ and $y$ direction respectively.

The reference template is thus moved all over the search image and, to evaluate their level of similarity, the OpenCV function cv2 . matchTemplate is used with the FNCC (Fast Normalized Cross-Correlation) criterion. The maximum value of the FNCC index is the one which detects the position of the template on the search image. Finally, the difference between the position of the template central pixel on the reference image and that on the search image yields the in-plane displacement.

In order to obtain the full-field maps, the displacements are computed at each point of the grid by sliding the reference template all over the search image. Once the displacements are evaluated, the strains are computed as a numerical differentiation process of the estimated displacements.

The software allows to perform the analysis in several steps by processing a stack of images. During the elaboration, the results are computed as the accumulated displacements and strains for each step of calculation and they are reported into text files for every pair of the processed images. At the end of the processing, the displacement and strain maps, the displacement quiver plot and the displacement plot related to the central section of the sample are shown and saved (Ravanelli et al., 2017).

\section{PY2DIC AND VIC-2D COMPARISON}

In order to evaluate the potentialities of py2DIC, tensile tests were performed in the Laboratory of Structural Engineering of the University of Rome "La Sapienza". Specifically, three different Glass Fiber Reinforced Polymer (GFRP) samples were subjected to tensile tests by means of a universal testing machine. During the tests, several strain gauges were fixed on the samples to measure strains and a Canon EOS 1200D camera was used to record images at different stages of deformation. The images were processed with py2DIC and the obtained results were compared with the values independently computed by the Vic-2D software. The horizontal (u) and vertical (v) displacements were calculated in millimetres for a pair of images; the obtained full-field displacement maps are shown in Figure 2 and Figure 3.

Regarding the $\mathrm{v}$ displacement fields, it is worth noticing that the sign of the y axis changes because of the different $y$ axis orientations in the two software. As a matter of fact, the $y$ axis is positive downwards in py2DIC while it is positive upwards in Vic-2D.

It is possible to observe a very good agreement at the level of one hundredth of millimetre between the $\mathrm{u}$ and $\mathrm{v}$ full-field displacement maps computed by py2DIC and those generated using Vic-2D. These first results hence highlight the quite good performances of FOSS py2DIC compared to the high cost commercial software Vic-2D.

\section{CONCLUSIONS AND PROSPECTS}

A new free and open source software, named py2DIC, was developed at the Geodesy and Geomatics Division of DICEA of the University of Rome "La Sapienza". In order to evaluate the potentialities of the developed software, the py2DIC performances were compared with the commercial software Vic-2D.

Specifically, the images acquired during tensile tests, performed in the Laboratory of Structural Engineering of the University of Rome "La Sapienza", were processed through the open source and the commercial software in order to obtain the horizontal $(\mathrm{u})$ and vertical (v) displacements. Py2DIC results are found to be in quite good agreement with those computed by Vic-2D, pointing out the potentialities of the developed FOSS package. As a future work, it could be worth using other sets of images to compare py2DIC performances with those of both commercial and open source DIC software. Specifically, full-field displacement and strain maps could be compared and standard statistical parameters could be computed in order to summarize the error of full-field displacements and strains of the different software. Besides, as the testing setup used to acquire the images is generally elementary, the aim of py2DIC project is to bring the methodology and the FOSS at a full 2D operational level in the widest practical cases, releasing as much as possible the acquisition constraints. Finally, the evolution from $2 \mathrm{D}$ to $3 \mathrm{D}$ analysis is also considered for the next future.

\section{ACKNOWLEDGEMENTS}

The authors are indebted with Prof. Nicola Nisticò and the Laboratory of Structural Engineering of University of Rome "La Sapienza" for the valuable assistance during the tensile experiments.

\section{REFERENCES}

Baqersad, J., Poozesh, P., Niezrecki, C. and Avitabile, P., 2017. Photogrammetry and optical methods in structural dynamics a 


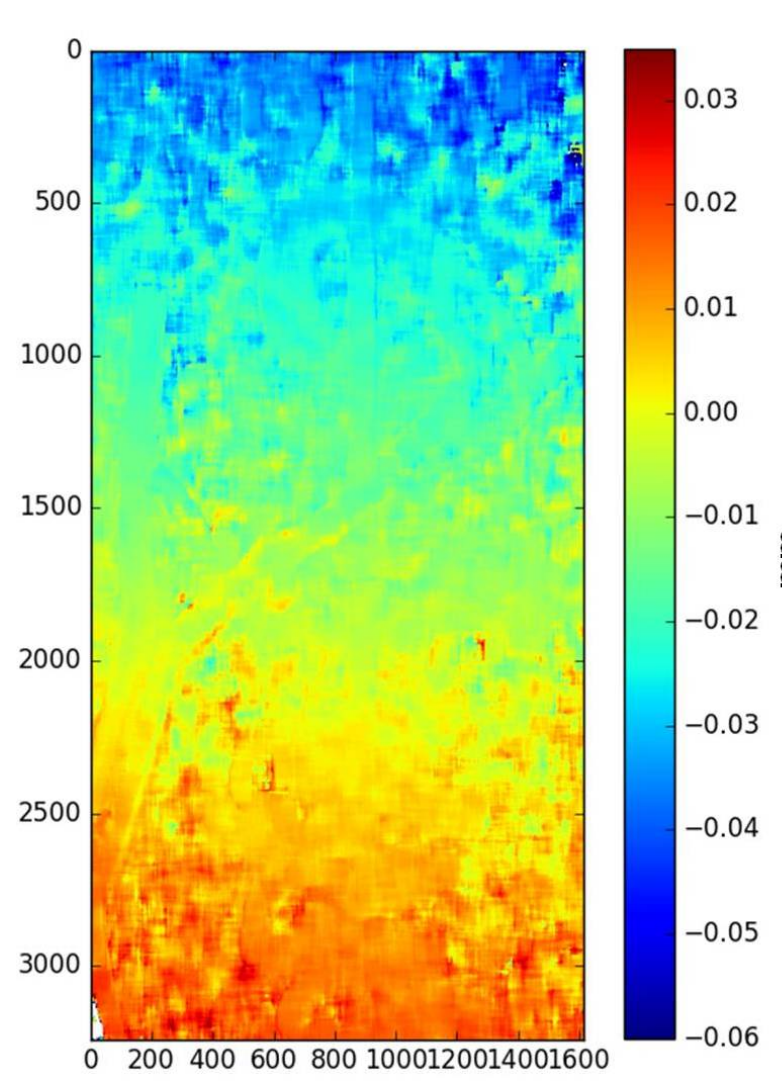

(a)

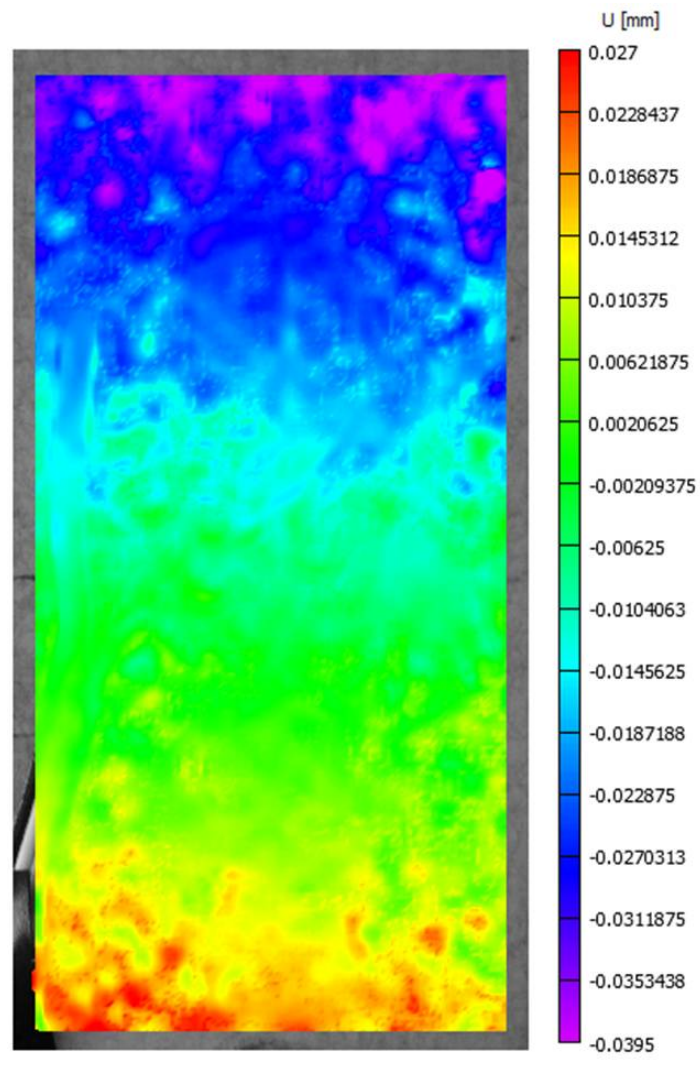

(b)

Figure 2: u displacement for the tensile test of GFRP sample obtained from DIC technique. (a) From py2DIC (b) From Vic-2D.

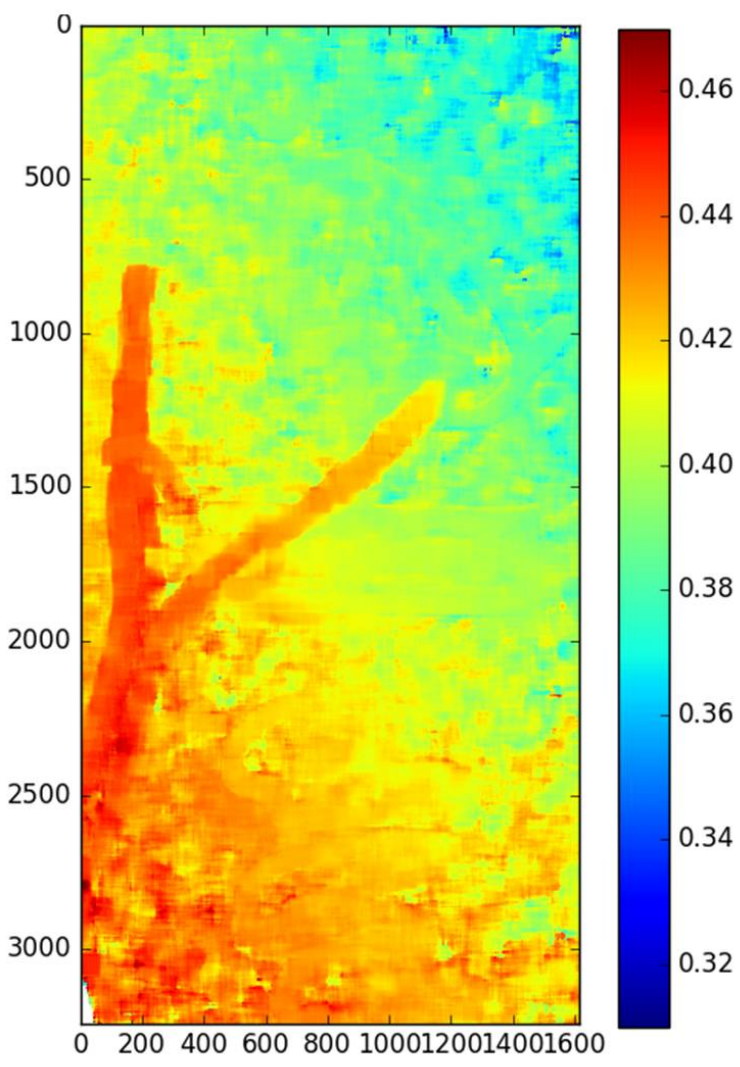

(a)

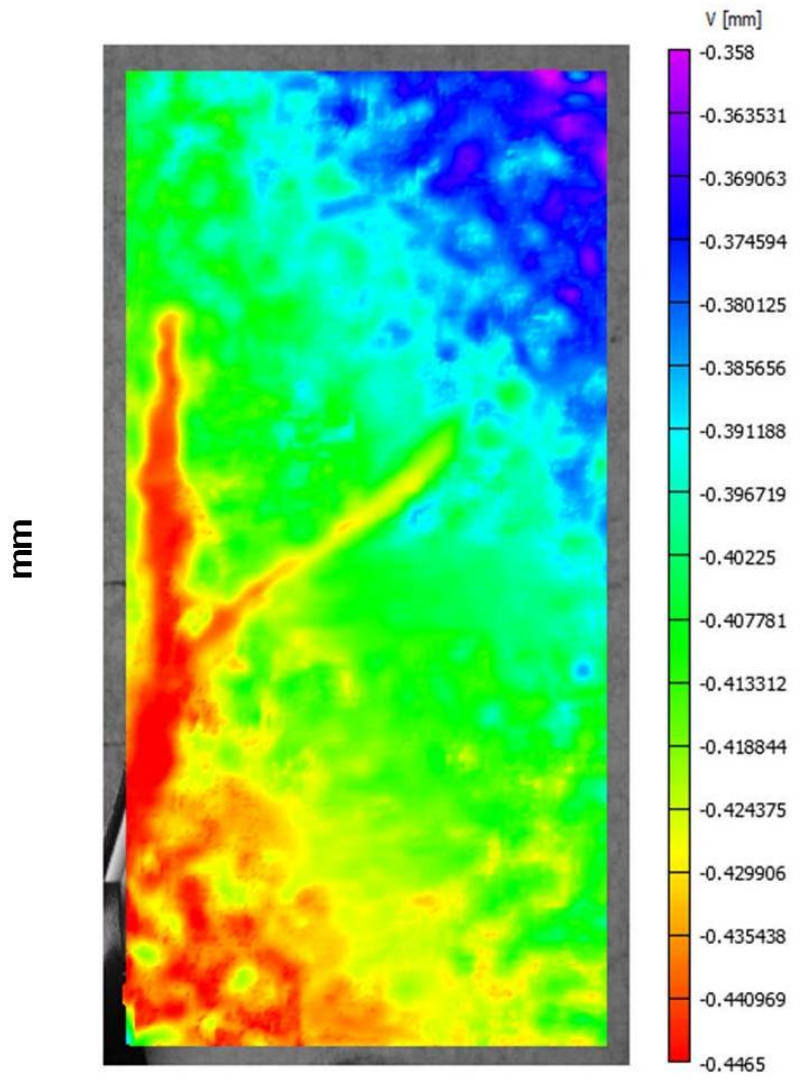

(b)

Figure 3: v displacement for the tensile test of GFRP sample obtained from DIC technique. (a) From py2DIC (b) From Vic-2D. 
review. Mechanical Systems and Signal Processing 86, pp. 17 - 34. Full-field, non-contact vibration measurement methods: comparisons and applications.

Belloni, V., 2017. A new Digital Image Correlation software for displacements field measurement in structural applications. M.S. Thesis, University of Rome "La Sapienza", Faculty of Civil and Industrial Engineering.

Blaber, J., Adair, B. and Antoniou, A., 2015. Ncorr: Open-source $2 \mathrm{~d}$ digital image correlation matlab software. Experimental Mechanics 55(6), pp. 1105-1122.

Bradski, G. and Kaehler, A., 2008. Learning OpenCV: Computer vision with the OpenCV library. O'Reilly Media, Inc.

Correlated Solutions, 2018. http://correlatedsolutions . com.

CorreliSTC, 2018. http://www. correli-stc.com.

Dantec Dynamics, 2018. https://www.dantecdynamics . com/digital-image-correlation.

gom, 2018. https://www.gom.com/metrology-systems/ aramis.html.

Harilal Ramesh and Ramji M, 2014. Adaptation of open source $2 \mathrm{~d}$ dic software ncorr for solid mechanics applications. pp. - .

Image Systems, 2018. http://www.imagesystems.se/ index.php.

Imetrum, 2018. https://www. imetrum. com/products.

Jones, E. M. C., Silberstein, M. N., White, S. R. and Sottos, N. R., 2014. In situ measurements of strains in composite battery electrodes during electrochemical cycling. Experimental Mechanics 54(6), pp. 971-985.

LaVision, 2018. https: //www . imetrum. com/products.

MatchID, 2018. http://www . matchidmbc.be.

McCormick, N. and Lord, J., 2010. Digital image correlation. Materials Today 13(12), pp. 52 - 54.

Ncorr, 2018. http://www.ncorr.com.

Pan, B., Qian, K., Xie, H. and Asundi, A., 2009. Twodimensional digital image correlation for in-plane displacement and strain measurement: a review. Measurement science and technology 20(6), pp. 062001.

Ravanelli, R., Nascetti, A., Di Rita, M., Belloni, V., Mattei, D., Nisticó, N. and Crespi, M., 2017. A new digital image correlation software for displacements field measurement in structural applications. ISPRS - International Archives of the Photogrammetry, Remote Sensing and Spatial Information Sciences XLII-4/W2, pp. 139-145.

Seghir, R., W. J. and Coudert, S., 2014. Yadics - Digital image correlation 2/3D software. http://www yadics. univ-lille1.fr/wordpress.

Turner, D., 2015. Digital Image Correlation Engine (DICe) Reference Manual. SAND2015-10606 O.

W. H. Peters, W. F. R., 1982. Digital imaging techniques in experimental stress analysis. Optical Engineering 21, pp. $21-21-$ 5. 